\title{
ATROPELLAMIENTO DE VERTEBRADOS EN TRES TIPOS DE CARRETERA DE LA REGIÓN MONTAÑOSA CENTRAL DE VERACRUZ, MÉXICO
}

\section{VERTEBRATE ROADKILLS IN THREE ROAD TYPES IN THE CENTRAL MOUNTAINOUS REGION OF VERACRUZ, MEXICO}

\section{Rodolfo CERVANTES-HUERTA, ${ }^{1}$ Federico ESCOBAR, ${ }^{1, *}$ JuAn Héctor GARCÍA-CHÁVEZ ${ }^{2}$ y Alberto GONZÁLEZ-ROMERO ${ }^{1}$}

\author{
${ }^{1}$ Instituto de Ecología, A. C., Carretera Antigua a Coatepec 351, Colonia El Haya, Xalapa 91070, Veracruz, \\ México. \\ ${ }^{2}$ Escuela de Biología, Benemérita Universidad Autónoma de Puebla, Puebla, México. \\ * Autor para correspondencia: <federico.escobarf@gmail.com>. \\ Recibido: 06/10/2016; aceptado: 01/06/2017. \\ Editor responsable: Vinicio Sosa.
}

Cervantes-Huerta, R., Escobar, F., García-Chávez, J. H. y González-Romero, A. (2017) Atropellamiento de vertebrados en tres tipos de carretera de la región montañosa central de Veracruz, México. Acta Zoológica Mexicana (n.s.), 33(3), 472-481.

RESUMEN. Este estudio evalúa la influencia del tipo de carretera y de la infraestructura (obras de drenaje) sobre el número de vertebrados atropellados en la carretera Xalapa - Puebla (tramo: Banderilla - Amozoc) en la zona central montañosa del Estado de Veracruz. El estudio se realizó en tres diferentes tipos de carretera (que varían de acuerdo al número de carriles y tipo de estructura que los separa) en los cuales se seleccionaron sitios donde existiera una obra de drenaje considerada como paso potencial para la fauna. En cada uno de los sitios se contó el número de individuos atropellados y se relacionó con la presencia - ausencia de obras de drenaje, la pendiente del derecho de vía con respecto a la carretera, así como el tipo de carretera. Se encontró un mayor número individuos atropellados en la supercarretera seguido por la autopista dividida por barrera central vegetativa y la autopista con barrera central de muro de concreto, y no se detectó un efecto de la presencia de las obras de drenaje sobre el número de individuos atropellados. Además, se encontró una relación negativa entre el número de individuos atropellados y la inclinación del derecho de vía. Este estudio contribuye a entender los factores que limitan el paso de vertebrados en las carreteras y discute sobre las implicaciones de la planeación-construcción de la infraestructura vial en el contexto de la conservación de la fauna silvestre.

Palabras clave: conservación, mortalidad de fauna, movilidad de fauna, obras de drenaje, tipo de carretera, topografía.

\section{INTRODUCCIÓN}

La infraestructura vial, en particular la red de carreteras, es una característica predominante de los paisajes de uso humano. En general, los países dedican hasta $2 \%$ de su
Cervantes-Huerta, R., Escobar, F., García-Chávez, J. H., \& González-Romero, A. (2017) Vertebrate roadkills in three road types in the central mountainous region of Veracruz, Mexico. Acta Zoológica Mexicana (n.s.), 33(3), 472-481.

ABSTRACT. This study evaluates the influence of the road type and infrastructure (culverts) on the numbers of vertebrates killed on the Xalapa - Puebla highway (section: Banderilla-Amozoc) in the central mountainous zone of the state of Veracruz. The study was carried out on three different road types (varying according to the number of lanes and type of structure that separates the lanes) along which sites were selected where there was a culvert considered as a potential wildlife crossing. Roadkills at each site were counted and related to the presence - absence of culverts, the slope of the right of way with respect to the road, as well as the road type. A greater number of roadkills occurred in the two-lanes highway followed by the highway divided by central vegetation barrier and the highway with central barrier of concrete wall. In none of the types of road studied were found an effect of the presence of culverts on the number of vertebrates killed. In addition, a negative relationship was found between the number of roadkills and the slope of the right of way. This study helps to understand the factors that limit the crossing of vertebrates on the roads and discusses the implications of road infrastructure planning - construction for wildlife.

Key words: Conservation, road infrastructure, road type, topography, wildlife mobility, wildlife mortality.

territorio a las carreteras y su derecho de vía (Forman, 1998) y de acuerdo con Forman y Deblinger (2000) los efectos de las carreteras se pueden extender hasta en un $20 \%$ del territorio como consecuencia de la fragmentación de los ecosistemas, afectando drásticamente la cantidad y 
la calidad de los hábitats disponibles. El desarrollo vial sostenido representa una de las mayores amenazas para la conservación de la biodiversidad con consecuencias para el funcionamiento de los ecosistemas (Geneletti, 2003).

Las carreteras limitan la movilidad de los organismos, dividen y aíslan las poblaciones (Forman 1995; Epps et al., 2005, 2007), lo cual tiene efectos sobre la diversidad de las comunidades, la dinámica poblacional y el intercambio genético (Taylor et al., 1993; Forman, 1998; Spellerberg, 1998; Epps et al., 2005). La fragmentación de los ecosistemas provocada por la construcción de carreteras crea una zona de transición o borde entre el área perturbada y el hábitat remanente. El efecto de borde de la carretera también afecta al microclima produciendo cambios en la temperatura y la humedad, velocidad del viento, y los niveles de radiación solar que puede influir directamente en la fauna silvestre o de forma indirecta a través de los cambios en la estructura y composición de la vegetación contigua a la carretera (Matlack, 1994).

Los ecólogos de carreteras reconocen los atropellamientos como uno de los factores más importantes de mortalidad de vertebrados que amenaza la dinámica y persistencia de las poblaciones (Havlick, 2004; SmithPatten \& Patten, 2008). Para especies con distribución restringida o para poblaciones aisladas, el atropellamiento, sumado a las afectaciones ocasionadas por el ruido, las vibraciones, la contaminación y el efecto barrera, puede llevar a la reducción de las poblaciones y a su extinción local (Havlick, 2004). Por lo tanto, si se espera mitigar el impacto de la construcción de carreteras sobre la vida silvestre es de gran importancia entender las implicaciones ecológicas de la creciente e inevitable red de caminos (Smith-Patten \& Patten, 2008). En este sentido, existe poca información sobre el efecto del tipo de carretera en la mortalidad de vertebrados y el papel de las obras de drenaje como potenciales pasos para la fauna.

La región de las altas montañas en la parte central del Estado de Veracruz es de gran importancia por su biodiversidad y servicios ecosistémicos (Williams-Linera et al., 2007), además de ser considerada una de las zonas de mayor diversidad florística de México (Rzedowski, 1991). En esta región se distribuyen un gran número de especies de vertebrados, algunas de las cuales son consideradas endémicas (NOM-059-SEMARNAT-2010). En los últimos 30 años el Estado de Veracruz ha experimentado un incremento importante de la infraestructura vial $(983 \%$ de acuerdo a INEGI 1987, 2015). El objetivo del estudio es evaluar la influencia de algunos factores considerados como determinantes para el paso de vertebrados en la carre- tera Xalapa - Puebla (en el tramo: Banderilla-Amozoc), una de la principales vías de comunicación entre el puerto de Veracruz y la Ciudad de México. Específicamente se abordan las siguientes preguntas: 1) ¿Cuál es el efecto de tres diferentes tipos de carretera (supercarretera sin barrera central, autopista con barrera central vegetativa y autopista con barrera central de muro de concreto) sobre la mortalidad de vertebrados por atropellamiento, 2) ¿De qué manera influye la presencia de las obras de drenaje (como potenciales pasos) sobre el atropellamiento de vertebrados? y, 3) ¿De qué manera se relacionan los atropellamientos de la fauna con la inclinación del derecho de vía de la carretera?

\section{MATERIALES Y MÉTODOS}

Área de estudio. El estudio se realizó entre los kilómetros $90+080$ al $116+330$ de la carretera federal 140D Xalapa - Puebla (tramo: Banderilla - Amozoc). Este tramo carretero sigue una dirección Suroeste - Noreste y abarca los municipios de Perote, Villa Aldama y Las Vigas de Ramírez en la región capital del Estado de Veracruz, México, entre las coordenadas UTM 2171647 al Norte, 2159942 al Sur, 667427 al Oeste y 695523 al Este (Fig. 1). Según datos proporcionados por la empresa Concesionaria $\mathrm{Au}-$ topista Perote Xalapa, S.A. de C.V. (COPEXA) para el año 2015 el aforo teórico vehicular promedio fue de 6,295 $\pm 1,482$ vehículos al día y un máximo de 13,041 vehículos al día, siendo diciembre el mes con mayor tráfico (238 mil vehículos/mes) y el mes de febrero con el menor tráfico (160 mil vehículos/mes). La velocidad máxima permitida a lo largo de toda la carretera es de $110 \mathrm{~km} / \mathrm{h}$.

A lo largo del tramo de carretera estudiado es posible distinguir tres tipos de carreteras, las cuales atraviesan diferentes ambientes: 1) la supercarretera Perote - Cantona - Puebla de tipo A2 (a la que se nombra supercarretera) de dos carriles y acotamiento de 2.5 metros a cada lado de la carretera con un ancho de calzada de 12 metros y derecho de vía de 60 metros y el cuerpo de la carretera fuera del centro de éste para una posterior ampliación a una autopista de tipo A4; este tramo atraviesa una zona de malpaís (derrame basáltico) originado por un derrame de lava del volcán Xalapaxquillo en el cual se desarrolla un matorral xerófilo (rosetófilo); 2) libramiento de Perote (a la que se nombra autopista con vegetación), es una autopista tipo A4 con ambos sentidos separados por una barrera central vegetativa de 12 metros sobre terreno plano y acotamiento de 2.5 metros al lado derecho de cada 


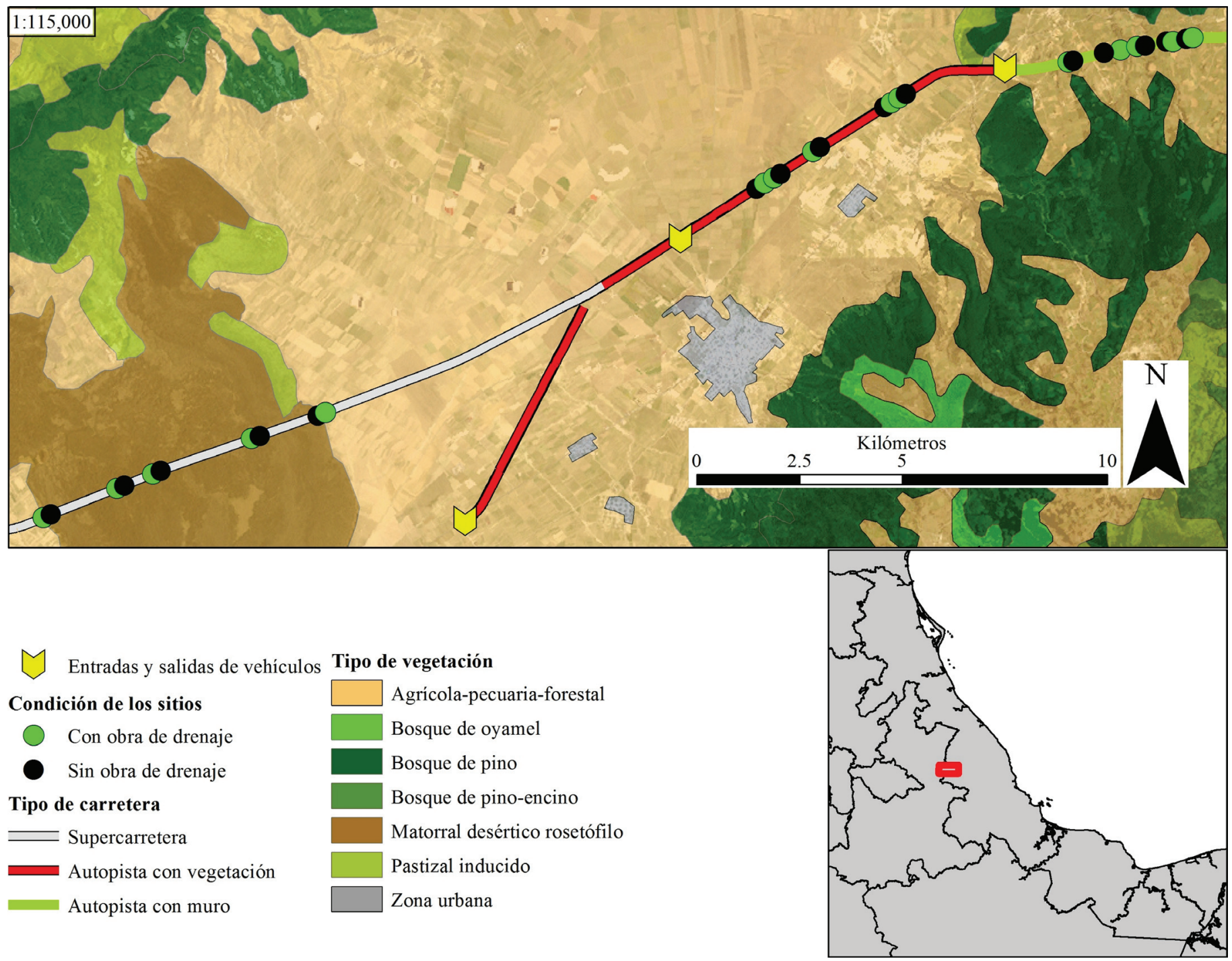

Figura 1. Área de estudio y ubicación del sitio de muestreo a lo largo de la carretera Xalapa-Puebla (tramo: Amozoc-Banderilla) en zona central del Estado de Veracruz, México.

sentido y derecho de vía de 60 metros, la cual atraviesa una zona de cultivo considerado como desprovisto de vegetación arbórea a excepción de algunas pequeñas áreas en las que se ha sembrado pino y 3 ) la autopista Banderilla - Perote de tipo A4 (a la que se nombra autopista con muro), con ambos sentidos separados por una barrera central de concreto sobre terreno montañoso con ambos sentidos separados con acotamiento de 2.5 metros al lado derecho de cada sentido y derecho de vía de 60 metros, la cual atraviesa áreas de bosque de pino fragmentado con zonas de cultivo.

En los tres tipos de carretera se construyeron obras de drenaje (consideradas como paso para la fauna) que van desde alcantarillas de tubo de lámina de 1.05 metros de diámetro mínimo, alcantarillas con loza de concreto de hasta seis metros de ancho, así como alcantarillas tipo bóveda y puentes de tamaño variable.

Muestreo. Para evaluar si la frecuencia de atropellamientos de vertebrados en cada tipo de carretera estaba asociada a las obras de drenaje, en cada tipo de carretera se seleccionaron cinco sitios que contuvieran una de las obras de drenaje descritas anteriormente (para un total de 15 sitios con obra de drenaje). Pareado a cada uno de estos sitios y a una distancia mínima de 150 metros, se seleccionó un sitio sin obra de drenaje a manera de control (15 sitios sin obra de drenaje). En cada sitio se buscó 
individuos muertos sobre la carretera mediante recorridos a pie 50 metros antes y después del punto de ubicación de la obra de drenaje (para un total de 30 sitios x 100 metros $=3$ kilómetros de carretera) durante un mínimo de 20 minutos y un máximo de 50 minutos, dependiendo del tráfico, condiciones climáticas y de la cantidad de cadáveres por colectar. Se colectaron todos los individuos posibles para ser identificados con la ayuda de un experto. Aquellas muestras que no pudieron ser identificadas se registraron como individuos 'no identificados'. En cada visita se retiraron del asfalto los restos de los individuos atropellados para evitar su conteo en el muestreo siguiente. En cada uno de estos sitios se tomó como covariable la altura del derecho de vía cada 20 metros a cada lado de la carretera.

Dado que el volumen del tráfico varía a lo largo del tiempo y a que el movimiento de la fauna varía de acuerdo con los periodos de dispersión de juveniles durante la temporada de reproducción o como una respuesta al cambio estacional en la disponibilidad de recursos (SmithPatten \& Patten, 2008; Hobday \& Mintrell, 2008), cada sitio fue visitado tres veces, considerando las temporadas climáticas de la región: temporada de secas (marzo a mayo, muestreo: 22 al 31 de mayo de 2015), temporada de lluvias (junio a octubre, muestreo: 31 de agosto al 3 de septiembre de 2015) y temporada de nortes (frentes fríos provenientes del norte con algo de precipitación; noviembre a febrero, muestreo: 25 al 27 de noviembre de 2015). El número de días de visita en cada temporada de muestreo dependió de la disponibilidad de cadáveres encontrados sobre la carretera.

Análisis de datos. Para evaluar la influencia del tipo de carretera y la presencia de las obras de drenaje sobre el número de individuos atropellados se utilizaron modelos lineares mixtos generalizados con distribución de error Poisson y liga log (Crawley, 2007). El modelo completo considerado fue: el tipo de carretera con tres niveles (supercarretera, autopista con vegetación y autopista con muro) y la presencia de la obras de drenaje con dos niveles (con y sin obra de drenaje) como factores fijos, y la interacción entre ambos factores considerando el sitio como el factor aleatorio con cinco niveles. La simplificación del modelo completo se llevó a cabo por el método de la máxima verosimilitud (Crawley, 2007). Posteriormente, se realizó una prueba de contrastes para evaluar las diferencias entre los niveles de cada factor fijo que resultaran significativos en el modelo.

Para analizar la relación entre la pendiente del derecho de vía (talud) de cada lado de la carretera y el número de atropellamientos, la altura del derecho de vía se transformó a grados de inclinación utilizando el promedio de la altura del derecho de vía obtenidas en cada sitio de cada lado de la carretera. Para el análisis se consideraron los sitios en los que las pendientes de cada lado de la carretera fueran: a) ambas positivas (cortes) o b) ambas negativas (terraplenes). Ambos valores se promediaron y el valor obtenido se relacionó con el número de cadáveres encontrados sobre la carretera mediante un análisis de covarianza, en el cual la inclinación fue la variable continua y el tipo de talud el factor con dos niveles (corte y terraplén). Para el análisis se excluyeron los datos del puente Banco del Horno localizado en el kilómetro 114+800 sobre la autopista con muro y su control debido a que no presenta un corte o terraplén, y por lo tanto, el suelo natural no es interrumpido por la carretera. Todos los análisis fueron realizados con ayuda del software $\mathrm{R}$ versión 3.2.4 (R Core Team, 2016).

\section{RESULTADOS}

En los tres kilómetros de carretera muestreado se encontraron 664 individuos atropellados, de los cuales 54\% (356 cadáveres) correspondieron a la temporada de secas, $32 \%$ (212 cadáveres) a la temporada de lluvias y 14\% (96 cadáveres) a la temporada de nortes. Del total de cadáveres encontrados 121 individuos (18\%) fueron identificados a nivel de especie, los cuales corresponden a 28 especies de 17 familias (Cuadro 1). Del total de cadáveres registrados el $40 \%$ fueron roedores y Peromyscus maniculatus fue la especie más abundante con 23 individuos (Cuadro 1).

El tipo de carretera con más especies atropelladas fue la autopista con vegetación con 18 especies, seguidas por la supercarretera y la autopista con muro con $15 \mathrm{y}$ 10 especies, respectivamente. Sobre la supercarretera se encontraron 414 cadáveres, equivalente al $62 \%$ del total (Apéndice 1), mientras que en la autopista con muro se encontró el menor número de atropellamientos (65 individuos equivalente a 10\% del total, Apéndice 1).

Cabe destacar el registro de varias especies de interés para la conservación como la ardilla de tierra de Perote (Xerospermophilus perotensis) y el colorín sietecolores (Passerina ciris), por su mención en la Lista Roja de especies amenazadas de la International Union for Conservation of Nature and Natural Resources (IUCN) (2015). Mientras que el camaleón de montaña (Phrynosoma orbiculare), la culebra sorda mexicana (Pituophis deppei) y la culebra lineada de bosque (Thamnophis cyrtopsis) 


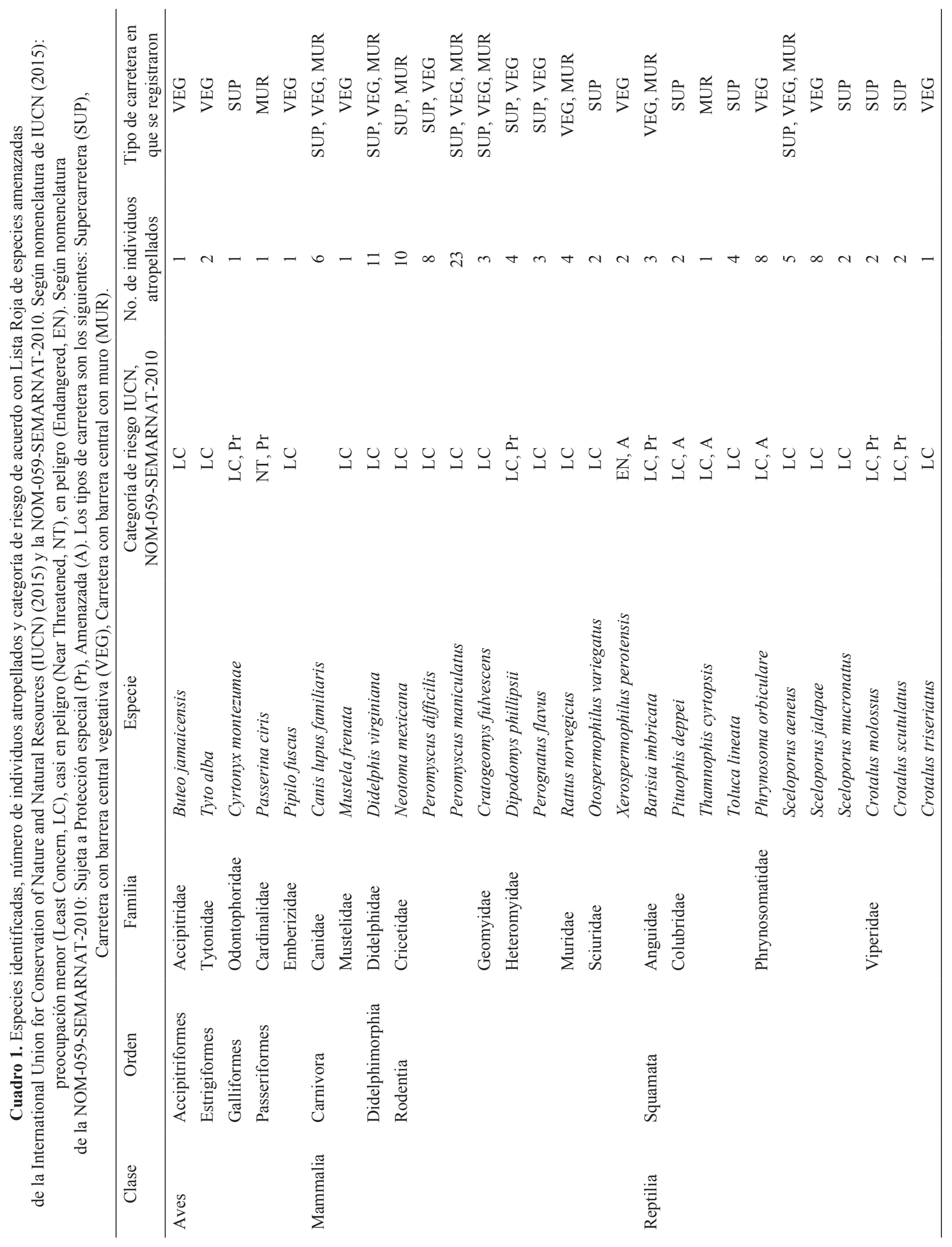


son consideradas como amenazadas (A) de acuerdo con la Norma Oficial Mexicana (NOM-059-SEMARNAT2010; Cuadro 1).

Influencia de la presencia de estructuras de drenaje y tipo de carretera. Ni la presencia de obras de drenaje ni su interacción con el tipo de carretera fueron importantes para explicar el número de individuos atropellados (Fig. 2A). La prueba de contrastes entre tipos de carreteras, mostró que el número promedio de cadáveres $( \pm \mathrm{EE})$ en la supercarretera $(41.4 \pm 0.67)$ fue significativamente mayor que el promedio en la autopista con vegetación (18.5 \pm $0.77, \mathrm{z}=7.18, \mathrm{P}<0.0001$ ), y este último fue significativamente mayor al promedio encontrado en la autopista con muro (6.5 $\pm 0.43, \mathrm{z}=7.78, \mathrm{P}<0.0001$, Fig. $2 \mathrm{~B})$. Es importante hacer notar que tanto en la supercarretera como en la autopista con vegetación se observó una mayor variación en el número de individuos atropellados por sitio en comparación con autopista con muro (Fig. 2B).

Influencia de la pendiente del límite del derecho de vía. El mayor número de individuos atropellados en los tres tipos de carretera fue encontrado en sitios en donde la inclinación del derecho de vía a ambos lados de la carretera fue menor a $20^{\circ}$ y esto fue más evidente sobre la supercarretera, en donde se encontraron entre 23 y 60 cadáveres por sitio (Fig. 3). En contraste, en la autopista con muro, cerca del $50 \%$ de los atropellamientos fueron encontrados en sitios en donde la inclinación del derecho de vía a ambos lados de la carretera fue mayor a los $20^{\circ}$. El análisis de covarianza mostró una relación negativa significativa entre el número de cadáveres y la inclinación del talud. Tanto la ordenada al origen como la pendiente de ambos tipos de talud fueron distintas de cero (terraplenes: $\mathrm{P}<0.0001$ y cortes: $\mathrm{P}=0.0167$ ) pero las pendientes de las curvas no fueron diferentes entre sí $(\mathrm{P}=0.8684$; Fig. 4).

\section{DISCUSIÓN}

Los resultados de este estudio indican que la mortalidad de fauna silvestre por colisión vehicular en el tramo Banderilla - Amozoc de la carretera Xalapa - Puebla es un problema preocupante (221 individuos atropellados/kilómetro), lo cual puede influir en la pérdida de diversidad de vertebrados y el mantenimiento de sus poblaciones en la región montañosa central del Estado de Veracruz (México), en particular para especies consideradas de importancia para la conservación. En este sentido, son necesarios los estudios que evalúen el efecto de las carreteras sobre el tamaño poblacional, concretamente para especies con distribución geográfica restringida o con alguna categoría de riesgo (X. perotensis, $P$. orbiculare, Barisia imbricata, Dipodomys phillipsii, NOM-059-SEMARNAT-2010).

En México no existen cifras a nivel nacional sobre la mortalidad de fauna a causa del atropellamiento vehicu-

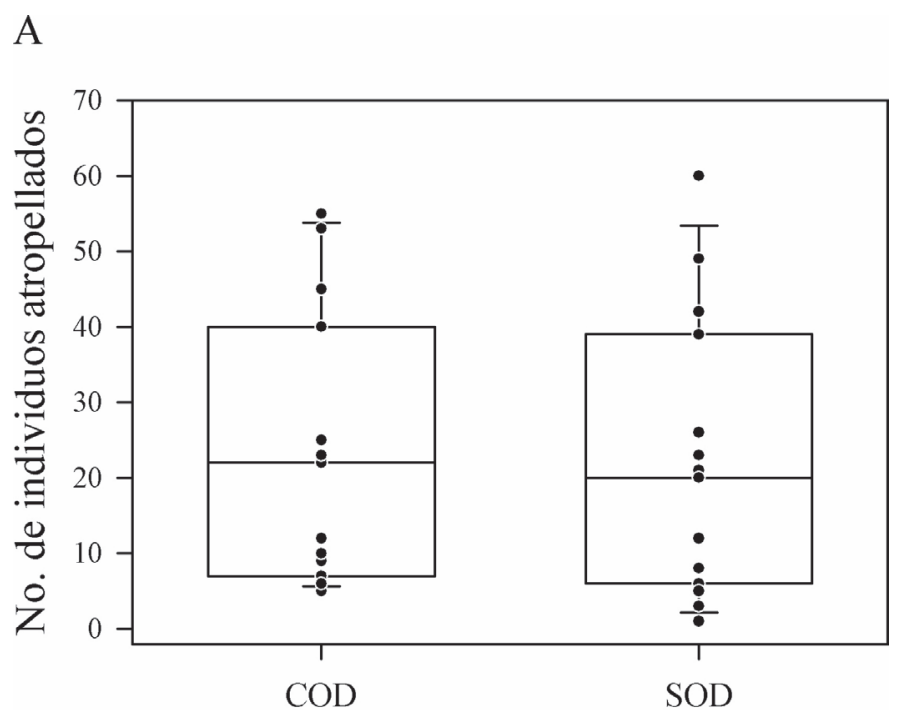

B

Condición

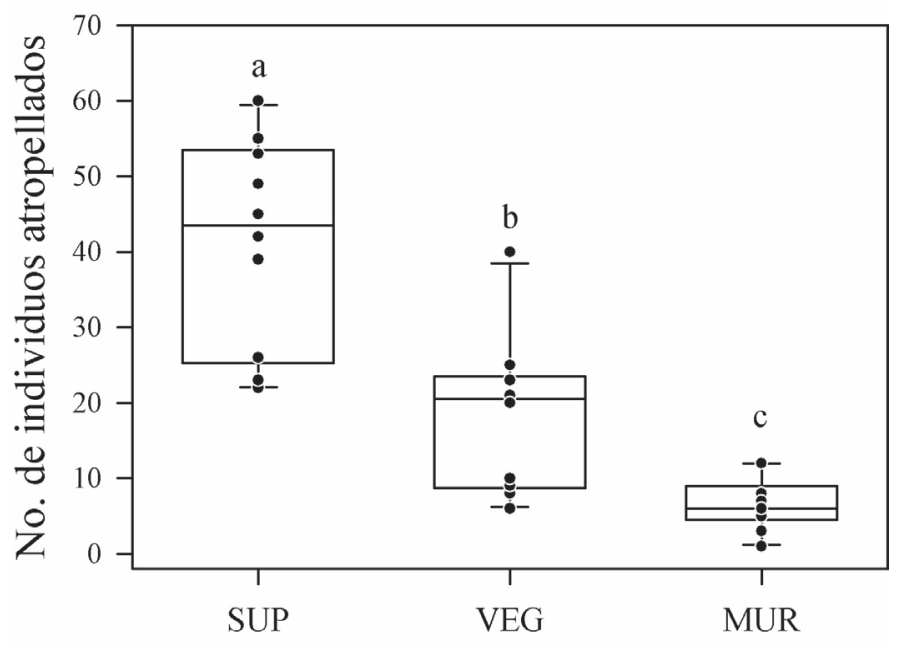

Tipo de carretera

Figura 2. Número de individuos atropellados sobre la carretera de acuerdo (A) presencia (COD) - ausencia (SOD) de obra de drenaje y (B) al tipo de carretera (SUP $=$ Supercarretera, $\mathrm{VEG}=$ Carretera con barrera central vegetativa, MUR = Carretera con barrera central con muro de concreto). La línea dentro de cada caja corresponde a la mediana, la altura de las cajas representan el primer y tercer cuartil, los bigotes son el valor máximo y mínimo. Los círculos negros representan los valores observados en cada sitio. Letras diferentes indican diferencias significativas. 


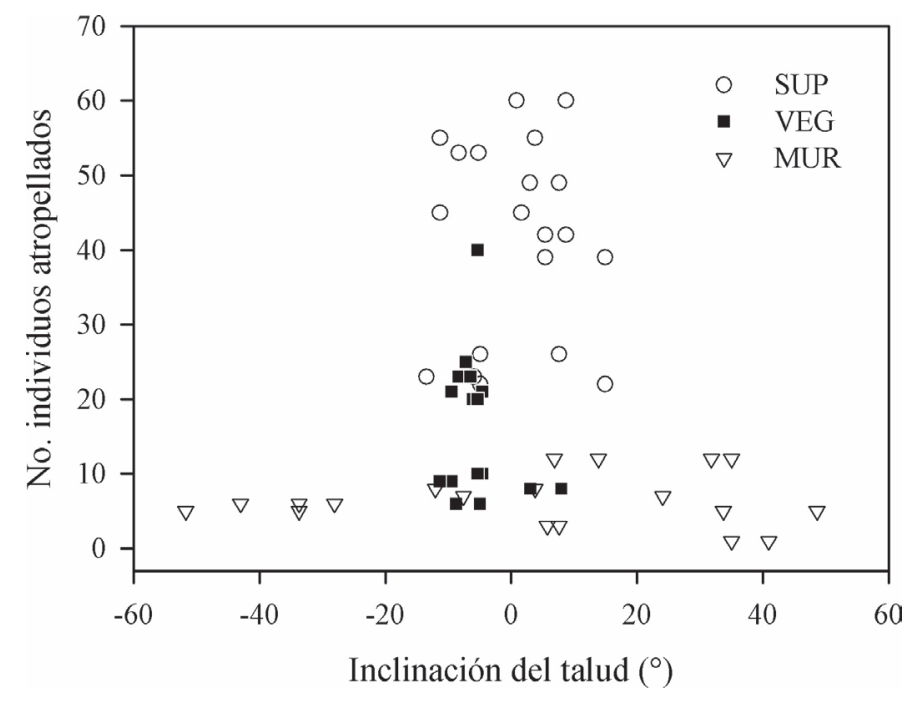

Figura 3. Número de individuos atropellados de acuerdo con la inclinación del talud en cada sitio a ambos lados de la carretera en cada tipo de carretera. Los valores negativos corresponden a la presencia de terraplenes y los valores positivos a la presencia de cortes. SUP $=$ Supercarretera, VEG $=$ Carretera con barrera central vegetativa, MUR $=$ Carretera con barrera central con muro de concreto.

lar lo cual impide tener una imagen sobre la magnitud de este problema en el país (Sánchez et al., 2013). De acuerdo con el Centro de Investigación para la Vida Silvestre se estima que en todas las autopistas de los Estados Unidos son atropellados diariamente cerca de millón de animales (Noss, 2002). Mientras que un estudio a lo largo de 20 años en España de la Sociedad para la Conservación de Vertebrados (SCV) se calcula en 30 millones (de 301 especies) el número de animales atropellados anualmente (PMVC 2003). Varios estudios confirman el efecto negativo de las carreteras sobre la abundancia de vertebrados (Shepard et al., 2008; Attademo et al., 2011; González-Gallina et al., 2013), aunque el impacto sobre un grupo taxonómico particular no solo depende del tipo de ecosistema y tipo de paisaje que la carretera atraviesa (Attademo et al., 2011), sino también de varios atributos inherentes a los rasgos de historia de vida de las especies, los cuales han sido relacionados con la vulnerabilidad a la colisión por vehículos, tales como el tamaño corporal (Adams \& Geis, 1983), el comportamiento de migración (e.g., anfibios; Fahrig et al., 1995), las preferencias de hábitat, la capacidad de desplazamiento y el rango hogareño (Carr \& Fahrig, 2001; Jedrzejewski et al., 2004).

Al igual que lo encontrado por Fahrig y Rytwinski (2009), en este estudio los mamíferos fue el grupo más afectado, seguido por el de los reptiles. En general, las carreteras rompen la continuidad de la vegetación e interrumpen la movilidad de los individuos y este efecto se incrementa según el ancho de la misma (Rondinini \& Doncaster, 2002), tal y como también fue observado en este estudio. Las carreteras al actuar como barrera pueden interceptar las rutas de movimiento y restringir la habilidad de los individuos de encontrar sus parejas, lo que puede llevar a la pérdida de su potencial reproductivo. De acuerdo con Carr y Fahrig (2001), esto podría ser una desventaja para especies con mayor movilidad y mayor rango habitacional. Cambios en la densidad poblacional y patrones de movimiento de las especies (e.j., depredadores, dispersores de semillas, herbívoros) como resultado del incremento de la densidad de carreteras en el paisaje puede tener consecuencias sobre la red trófica, afectando las interacciones entre especies y la funcionalidad de los ecosistemas que atraviesan (Forman \& Alexander, 1998; Trombulak \& Frissell, 2000; Ruiz-Capillas et al., 2013). Un tema que requiere de mayor atención por parte de los ecólogos dedicados al estudio de los impactos de las carreteras.

Los resultados indican que la autopista con muro puede considerarse como una barrera más efectiva para el cruce

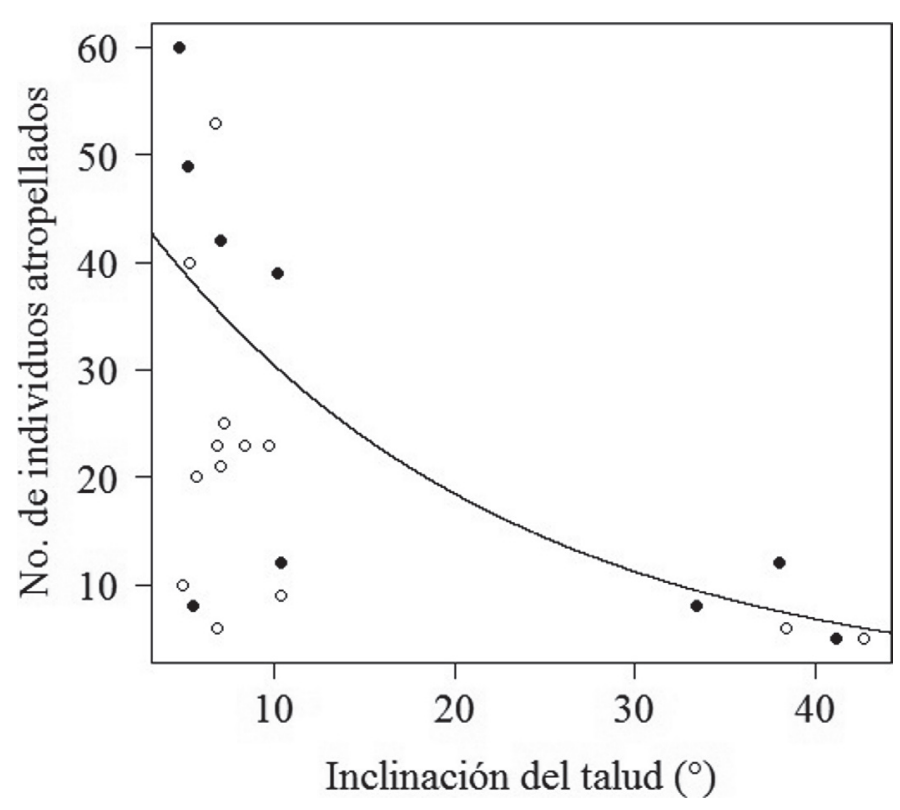

Figura 4. Relación entre el número de individuos atropellados sobre la carretera y la inclinación del talud de la carretera. Los puntos negros indican los sitios con cortes (valores positivos) y los puntos blancos indican los sitios con terraplenes (valores negativos) a ambos lados de la carretera. 
de la fauna silvestre que la autopista con vegetación y la supercarretera. Contrario a lo encontrado en este estudio, Smith-Patten y Patten (2008) en el centro de Estados Unidos no encontraron diferencias entre la autopista con barrera central de concreto y la autopista con barrera central vegetativa. Mientras que Taylor y Goldingay (2004) en el sureste de Australia, observaron un mayor número de cadáveres en una carretera de dos carriles comparado con una carretera de cuatro carriles. Esto confirma que en cuanto más ancho el camino, mayor es el efecto barrera y menor la conectividad entre lados opuestos de la carretera.

El tipo de estructura que separa los carriles de una carretera es otro factor que influye en la probabilidad de cruce de la fauna (Smith-Patten \& Patten, 2008). Para el caso de carreteras con barrera central vegetativa el paso de fauna podría ser favorecido, debido a que estas pueden servir como áreas de descanso, actuando como 'piedra de paso' para moverse entre lados opuestos de la carretera (SmithPatten \& Patten, 2008). Por el contrario, en carreteras con barrera central de concreto, dicha estructura es otro de los obstáculos que la fauna silvestre debe sortear que acentúa el efecto de barrera de la carretera. Esto explica por qué el cruce de la fauna silvestre en esta carretera fue menor en comparación con la autopista con vegetación y la supercarretera. Sin embargo, es necesario considerar otros factores como el flujo vehicular y la cercanía a poblados, lo cual incrementa la contaminación lumínica y el ruido, los cuales modifican el comportamiento de las especies en áreas aledañas a las carreteras e incrementan la probabilidad de atropellamiento (Forman \& Alexander, 1998).

En ninguno de los tres tipos de carreteras estudiadas se tuvo en cuenta el diseño y construcción de pasos adecuados para la fauna silvestre. En su lugar, se decidió considerar a los puentes y obras de drenaje como sitios para el paso de la fauna, lo que podría ser conveniente si estas obras se construyeran en lugares apropiados, de manera adecuada y en cantidad suficiente a lo largo de la carretera (Reséndiz et al., 2011). Los resultados de este estudio muestran que las obras de drenaje no contribuyen de forma efectiva al paso de la fauna de vertebrados ya que su presencia no redujo el número de individuos atropellados sobre la vía. Por lo tanto, es necesario la implementación de protocolos de monitoreo adecuados para cada grupo taxonómico para evaluar la efectividad de las obras de mitigación como pasos para la fauna silvestre.

Pocas investigaciones consideran las características topográficas del terreno para evaluar el impacto de las carreteras sobre el cruce de la fauna silvestre. Los resultados de este estudio indican que los sitios con pendientes del derecho de vía (taludes) con menos de $20^{\circ}$ de inclinación concentraron el mayor número de cadáveres sobre la carretera. La construcción de carreteras sobre relieve montañoso obliga a los constructores a realizar cortes y construir terraplenes en el terreno (TÜV et al., 2000) que actúan como una barrera física para la fauna, lo que aumenta el efecto de borde y por lo tanto de barrera para la fauna, tal y como fue observado en la autopista con muro. En este sentido, es necesaria la construcción de obras que, además de restringir el paso en ciertos tramos de la carretera, dirijan a la fauna silvestre a lugares de paso seguro, lo cual podría reducir la probabilidad de colisión.

\section{CONCLUSIONES}

Contrario a lo que se esperaba, este estudio muestra que la presencia de obras de drenaje como potenciales pasos para la fauna no tuvo influencia sobre el número de individuos de vertebrados atropellados en la carretera. No solo el ancho de la carretera, sino el tipo de estructura central que divide los carriles tuvieron un fuerte efecto sobre la mortalidad de fauna. Además, la inclinación de talud (i.e., altura y pendiente del derecho de vía) en sinergia con el tipo de estructura que divide los carriles (e.j., carretera con muro) al parecer actúa como una barrera más efectiva para el movimiento de la fauna silvestre. Los resultados de este estudio ponen de manifiesto la importancia de estudios que cuantifiquen el atropellamiento de vertebrados en carreteras que atraviesan diferentes ecosistemas. Esto resulta de gran importancia dado el considerable incremento urbanístico y de la red vial en las últimas décadas en México (Garza, 2010). El incremento de la densidad de carreteras es sin duda un factor que contribuye a la pérdida de fauna y modifica la estructura y dinámica poblacional de vertebrados, en particular de especies con distribución geográfica restringida y de interés para la conservación.

\section{LITERATURA CITADA}

Adams, L. W., \& Geis, A. D. (1983). Effects of roads on small mammals. Journal of Applied Ecology, 20, 403-415.

Attademo, A. M., Peltzer, P. M., Lajmanovich, R. C., Elberg, G., Junges, C., Sánchez L. C. \& Bassó, A. (2011). Wildlife vertebrate mortality in roads from Santa Fe Province, Argentina. Revista Mexicana de Biodiversidad, 82, 915-925.

Carr, L. W. \& Fahrig, L. (2001). Effects of road traffic on two amphibian species of differing vagility. Conservation Biology, 15, 1071-1078. 
Crawley, M. J. (2007). The R book. John Wiley \& Sons Ltd., England, $942 \mathrm{p}$.

Epps, C. W., Palsboll, P. J., Wehausen, J. D., Bleich, V. C., Torres, S. G. \& Brashares, J. S. (2007). Optimizing dispersal and corridor models using landscape genetics. Journal of Applied Ecology, 44, 714-724.

Epps, C. W., Palsboll, P. J., Wehausen, J. D., Roderick, G. K., Ramey, R. R. I. I. \& McCullough, D. R. (2005). Highways block gene flow and cause a rapid decline in genetic diversity of desert bighorn sheep. Ecology Letters, 8, 1029-1038.

Fahrig, L., Pedlar, J. H., Pope, S. E., Taylor, P. D. \& Wegner, J. F. (1995). Effect of road traffic on amphibian density. Biological Conservation, 73, 177-182.

Fahrig, L. \& Rytwinski, T. (2009). Effects of roads on animal abundance: an empirical review and synthesis. Ecology and Society, 14, 21 Disponible en: <http://www.ecologyandsociety.org/vol14/ iss $1 /$ art $21 />$

Forman, R. T. T. (1995). Land Mosaics: The Ecology of Landscapes and Regions. Cambridge University Press, United Kingdom, 632 p.

Forman, R. T. T. (1998). Road ecology: A solution for the giant embracing us. Landscape Ecology, 13, III-V.

Forman, R. T. T. \& Alexander, L. E. (1998). Roads and their major ecological effects. Annual Review of Ecology and Systematics, 29, 207-231.

Forman, R. T. T. \& Deblinger, R. D. (2000). The ecological roadeffect zone for transportation planning, and a Massachusetts highway example. Conservation Biology, 14, 36-46.

Garza, G. (2010). La transformación urbana de México, 1970 - 2020. Pp. 31-86. En: Garza, G. \& Schteingart, M (Eds.). Desarrollo urbano y regional. El Colegio de México, México, D.F.

Geneletti, D. (2003). Biodiversity Impact Assessment of roads: an approach based on ecosystem rarity. Environmental Impact Assessment Review, 23, 343-365.

González-Gallina, A., Benitez-Badillo, G., Rojas-Soto, O. R. \& Hidalgo-Mihart, M. G. (2013). The small, the forgotten and the dead: highway impact on vertebrates and its implications for mitigation strategies. Biodiversity and Conservation, 22, 325-342.

Havlick, D. (2004). Roadkill. Conservation Magazine, 5, 30-34.

Hobday, A. J. \& Mintrell, M. L. (2008). Distribution and Abundance of Roadkill on Tasmanian Highways: Human Management Options. Wildlife Research, 35, 712-726.

IUCN. (2015). The IUCN Red List of Threatened Species. Version 2015-3. Disponible en: http://www.iucnredlist.org (Consultada en Agosto 2015).

INEGI (Instituto Nacional de Estadística y Geografía). (1987). Anuario Estadístico y Geográfico Por Entidad Federativa. Edición 1986. Disponible en: http://www3.inegi.org.mx/sistemas/biblioteca/ficha.aspx?upc=702825141479 (Consultada en Agosto 2015).

INEGI (Instituto Nacional de Estadística y Geografía). (2105). Anuario Estadístico y Geográfico Por Entidad Federativa. Edición 2014. Disponible en: http://www3.inegi.org.mx/sistemas/biblioteca/ficha.aspx?upc=702825077297 (Consultada en Agosto 2015).

Jedrzejewski, W., Niedzialkowska, M., Nowak, N., \& Jedrzejewska, B. (2004). Habitat variables associated with wolf (Canis lupus) distribution and abundance in northern Poland. Diversity and Distributions, 10, 225-233.
Matlack, G. R. (1994). Vegetation dynamics of the forest edge: trends in space and successional time. Journal of Ecology, 82, 113-123.

Noss, R. (2002). The ecological effects of roads. Disponible en http:// www.eco-action.org/dt/roads.html. (Consultada el 13 de mayo de 2017).

PMVC (Proyecto provisional de seguimiento de la mortalidad de vertebrados en carreteras). (2003). Mortalidad de vertebrados en carreteras. Documento técnico de conservación $n^{\circ} 4$. Sociedad para la Conservación de los Vertebrados (SCV), Madrid. $350 \mathrm{p}$.

R Core Team. (2016). R: A language and environment for statistical computing. R Foundation for Statistical Computing, Vienna, Austria. Available at: https://www.R-project.org/ (Consultada en Abril 2015)

Reséndiz, J. M., Pille, R. \& Flores, T. (2011). Efecto ambiental de caminos. Pp. 129 - 160. En: López, S. A \& Fernández N. (Eds.). La Gestión ambiental de carreteras en México. Grupo Selome, México, D. F.

Rondinini, C. \& Doncaster, C. P. (2002). Roads as barriers to movement for hedgehogs. Functional Ecology, 16, 504-509.

Ruiz-Capillas, P., Mata, C. \& Malo, J. E. (2013). Community response of mammalian predators and their prey to motorways: Implications for predator-prey dynamics. Ecosystems, 16, 617-626.

Rzedowski, J. (1991). El endemismo en la flora fanerogámica mexicana: una apreciación analítica preliminar. Acta Botánica Mexicana, 15, 47-64.

Sánchez, P. J. I., Delgado-Trejo, C., Mendoza-Ramírez, E. \& Sauzo-Ortuño, I. (2013). Las carreteras como una fuente de mortalidad de fauna silvestre de México. CONABIO. Biodiversitas, 111, 12-16.

SEMARNAT (Secretaría de Medio Ambiente y Recursos Naturales). (2010). Norma Oficial Mexicana NOM-059-SEMARNAT2010 Protección ambiental Especies nativas de México de flora y fauna silvestres - Categorías de riesgo y especificaciones para su inclusión, exclusión o cambio - Lista de especies en riesgo. Diario Oficial de la Federación, Segunda Sección.

Shepard, D., Kuhns, A., Dreslik, M. \& Phillips, C. (2008). Roads as barriers to animal movement in fragmented landscapes. Animal Conservation, 11, 288-296.

Smith-Patten, B. D. \& Patten, M. A. (2008). Diversity, seasonality, and context of mammalian roadkills in the southern Great Plains. Environmental Management, 41, 844-852.

Spellerberg, I. F. (1998). Ecological effects of roads and traffic: a literature review. Global Ecology and Biogeography, 7, 317-333.

Taylor, B. D. \& Goldingay R. L. (2004). Wildlife road-kills on three major roads in north-eastern New South Wales. Wildlife Research, 31, 83-91.

Taylor, P. D., Fahrig, L., Henein, K. \& Merriam, G. (1993). Connectivity is a vital element of landscape structure. Oikos, 68, 571573.

Trombulak, S. C. \& Frissell, C. A. (2000). Review of ecological effects of roads on terrestrial and aquatic communities. Conservation Biology, 14, 18-30.

TÜV Rheinland de México S.A. de C.V., Damián Hernández, S. A., Martínez Soto, A. \& Aguirre Pérez, J. M. (2000). Impacto ambiental de proyectos carreteros. Efectos por la construcción y operación del drenaje y subdrenaje. Publicación Técnica, 155. 
Williams-Linera, G., Guillén, A., Gómez O. \& Lorea, F. (2007). Conservación en el centro de Veracruz, México. El bosque de niebla: ¿reserva archipiélago o corredor biológico? Pp. 303-310. En: Halffter, G., Guevara, S. \& Melic, A. (Eds.). Hacia una cultura de conservación de la diversidad biológica. SEA, CONABIO, CONANP, CONACYT, INECOL, UNESCO-MAB y Ministerio del Medio Ambiente-Gobierno de España. Monografías Tercer Milenio, Vol. 6. Zaragoza, España.

\section{APÉNDICE 1}

Número de individuos y especies identificadas de vertebrados atropellados por tipo de carretera, sitio (kilómetros) y tipo de obra de drenaje. Supercarretera (SUP); autopista con vegetación (VEG); autopista con muro (MUR).

\begin{tabular}{|c|c|c|c|c|c|}
\hline Tipo de carretera & Sitio & Tipo de obra de drenaje & $\begin{array}{l}\text { Número de } \\
\text { individuos }\end{array}$ & Número de especies* & $\begin{array}{c}\text { Total individuos / } \\
\text { especies }\end{array}$ \\
\hline \multirow[t]{7}{*}{ SUP } & $90+130$ & Alcantarilla de tubo de concreto & 53 & 3 & $414 / 15$ \\
\hline & $92+054$ & Alcantarilla con losa de concreto & 45 & 2 & \\
\hline & $92+254$ & Sin estructura & 39 & 3 & \\
\hline & $95+563$ & Alcantarilla con losa de concreto & 55 & 0 & \\
\hline & $95+763$ & Sin estructura & 60 & 1 & \\
\hline & $97+265$ & Sin estructura & 42 & 0 & \\
\hline & $97+465$ & Alcantarilla con losa de concreto & 23 & 2 & \\
\hline \multirow{7}{*}{ VEG } & $105+435$ & Sin estructura & 21 & 1 & \\
\hline & $106+398$ & Alcantarilla con losa de concreto & 40 & 5 & \\
\hline & $106+598$ & Sin estructura & 20 & 0 & \\
\hline & $108+440$ & Sin estructura & 8 & 3 & \\
\hline & $108+640$ & Alcantarilla con losa de concreto & 9 & 1 & \\
\hline & $108+857$ & Puente & 10 & 3 & \\
\hline & $109+057$ & Sin estructura & 6 & 0 & \\
\hline \multirow[t]{5}{*}{ MUR } & $113+159$ & Alcantarilla de tubo de lámina & 5 & 0 & $65 / 10$ \\
\hline & $113+309$ & Sin estructura & 8 & 3 & \\
\hline & $115+780$ & Alcantarilla con losa de concreto & 12 & 1 & \\
\hline & $116+130$ & Sin estructura & 5 & 1 & \\
\hline & $116+280$ & Alcantarilla de tubo de concreto & 6 & 1 & \\
\hline
\end{tabular}

